\title{
Os fatores condicionantes ao pré-natal de alto risco: Revisão integrativa
}

\author{
The conditioning factors for high risk prenatal care: Integrative review \\ Los condicionantes para la atención prenatal de alto riesgo: Revisión integradora
}

Recebido: 06/11/2021 | Revisado: 12/11/2021 | Aceito: 23/11/2021 | Publicado: 02/12/2021

\author{
Eduardo Morais da Silva \\ ORCID: https://orcid.org/0000-0003-0159-0455 \\ Universidade Ceuma, Brasil \\ E-mail: enfermeiroedu12@gmail.com \\ Patrícia dos Santos Silva Queiroz \\ ORCID: https://orcid.org/0000-0002-9587-1786 \\ Universidade Ceuma, Brasil \\ E-mail: patriciasqueiroz@gmail.com \\ Jullys Allan Guimarães Gama \\ ORCID: https://orcid.org/0000-0002-8834-6326 \\ Universidade Ceuma, Brasil \\ E-mail: jullys.gama@gmail.com \\ Artur de Souza Veras \\ ORCID: https://orcid.org/0000-0002-6160-8736 \\ Universidade Estadual do Pará, Brasil \\ E-mail: lasferro1@gmail.com \\ Keiliane Pinheiro Silva Barros \\ ORCID: https://orcid.org/0000-0002-0477-9766 \\ Universidade Ceuma, Brasil \\ E-mail: keilianepinheiromk@gmail.com \\ Francisco Alves Lima Júnior \\ ORCID: https://orcid.org/0000-0002-3117-4949 \\ Universidade Ceuma, Brasil \\ E-mail: francisco.enfdotrabalho@gmail.com \\ Érika Ferreira Tourinho \\ ORCID: https://orcid.org/0000-0003-2167-930X \\ Universidade Ceuma, Brasil \\ E-mail: tourinhoerika@gmail.com
}

\begin{abstract}
Resumo
Introdução: O acompanhamento de pré-natal corresponde monitoramento da saúde das gestantes, a fim de risco com intuito de trazer suporte e qualidade de vida para mãe/feto. Objetivo: Conhecer os fatores condicionantes ao Pré-Natal de Alto Risco. Metodologia: Trata-se de revisão integrativa, também possui um método exploratório qualitativo. Resultados: Percebe-se que a gestante necessita de um acolhimento integral por especialistas, tendo direito a consultas individuais e grupo, de intervalos regulares até o parto. Esta assistência será principalmente para que as complicações rastreadas sejam sanadas até o nascimento do bebê. Observa-se que não somente a prevenção de riscos na gestação, fundamente-se que a promoção em saúde deve estar implementando visando acolhimento destas gestantes e um olhar mais singular, assim contribuindo para rastreamento de fatores condicionantes e educação em saúde destas mulheres. Considerações finais: Cabe à equipe multiprofissional, em especial a da atenção primária, conhecer os fatores de um pré-natal de alto risco e estar apta a identificar e acompanhar a fim de impedir maiores complicações, tomando ações que priorizem um atendimento especializado e humanizado, visando também a promoção do empoderamento das gestantes através de ações educativas em saúde com o intuito de reduzir as mortes em decorrência de um pré-natal de alto risco.
\end{abstract}

Palavras-chave: Saúde da mulher; Cuidado pré-natal; Gravidez de alto risco.

\begin{abstract}
Introduction: Prenatal monitoring corresponds to monitoring the health of pregnant women, in order to risk in order to bring support and quality of life to the mother/fetus. Objective: To know the conditioning factors for high-risk prenatal care. Methodology: This is an integrative review, it also has a qualitative exploratory method. Results: It is noticed that the pregnant woman needs full care by specialists, with the right to individual and group consultations, at regular intervals until delivery. This assistance will be mainly so that the traced complications are remedied until the baby's birth. It is observed that not only the prevention of risks during pregnancy, but also that health promotion should be implemented aiming at welcoming these pregnant women and a more unique look, thus contributing to the tracking of conditioning factors and health education for these women. Final considerations: It is up to the multidisciplinary team, especially that of primary care, to know the factors of high-risk prenatal care and be able to
\end{abstract}


identify and monitor in order to prevent further complications, taking actions that prioritize specialized and humanized care, also aiming to promote the empowerment of pregnant women through health education actions with the aim of reducing deaths due to high-risk prenatal care.

Keywords: Women's health; Prenatal care; Pregnancy high-risk.

\section{Resumen}

Introducción: El seguimiento prenatal corresponde al seguimiento de la salud de la gestante, con el fin de arriesgar con el fin de brindar apoyo y calidad de vida a la madre / feto. Objetivo: Conocer los condicionantes de la atención prenatal de alto riesgo. Metodología: Se trata de una revisión integradora, también cuenta con un método exploratorio cualitativo. Resultados: Se advierte que la gestante necesita una atención integral por parte de especialistas, con derecho a consultas individuales y grupales, en intervalos regulares hasta el parto. Esta asistencia será principalmente para que las complicaciones rastreadas se remedien hasta el nacimiento del bebé. Se observa que no solo la prevención de riesgos durante el embarazo, sino también que se debe implementar la promoción de la salud con el objetivo de acoger a estas gestantes y una mirada más singular. contribuyendo así al seguimiento de condicionantes y educación para la salud de estas mujeres. Consideraciones finales: Corresponde al equipo multidisciplinario, especialmente el de atención primaria, conocer los factores de la atención prenatal de alto riesgo y poder identificarlos y monitorearlos para prevenir mayores complicaciones, tomando acciones que prioricen la atención especializada y humanizada, también con el objetivo de promover el empoderamiento de las mujeres embarazadas a través de acciones de educación en salud con el fin de reducir las muertes por atención prenatal de alto riesgo.

Palabras chave: Salud de la mujer; Atención prenatal; Embarazo de alto riesgo.

\section{Introdução}

O cuidado a saúde da mulher é uma prioridade na saúde pública do Brasil por conta do histórico elevado de morbimortalidade materna, a taxa atual é de 64,5 óbitos para cada 100 mil nascidos vivos, sendo que $92 \%$ destas mortes são evitáveis no rastreamento no pré-natal (Campos et al., 2019) (Dalla Costa et al., 2016).

O acompanhamento de pré-natal corresponde monitoramento da saúde das gestantes, a fim de risco com intuito de trazer suporte e qualidade de vida para mãe/feto. O Pré Natal de Alto Risco é considerado pelo Ministério da Saúde (2012) o a qual a vida ou saúde da mãe e/ou do feto e/ou do recém-nascido têm maiores chances de serem atingidas que o restante da população.

As condições envolvidas para o prognostico prejudicial materno/fetal são individuais e estão associadas ao perfil socioeconômico, situação demográfica, histórico de gravidezes anteriores, estado clínico e obstétrico, alimentação e comorbidades que refletem no estado gravídico, levando a evolução de quadros como hipertensão arterial, diabetes mellitus, pré-eclâmpsia, eclampsia, síndrome de hellp, desnutrição, obesidade, infecções urinárias (Sanine et al., 2019).

Os dados epidemiológicos da gestação de alto risco correspondem de $15 \%$ do total de gestações, expressando 470 mil gestações ao ano no Brasil, seguido pelas principais patologias responsáveis pela Gravidez de Alto Risco, a prevalência da Diabetes Mellitus Gestacional passou a ser de 17,8\% do total de gestantes e Hipertensão Arterial Gestacional cerca de 7\% das grávidas brasileiras (Saúde, 2017) (Sánchez-Gomez et al., 2016).

A triagem de gestação de alto risco começa na atenção básica, sendo referenciado ao serviço especializado para o acompanhamento necessário disposto pela norma o Ministério de Saúde (2001) como Sistemas Estaduais de Referência Hospitalar a Gestante de Alto Risco. Por isso, além da detecção, deve-se trabalhar em conjunto para avaliação correta.

Diante disso, torna-se importante o levantamento dos fatores condicionantes relacionados ao atendimento dos prénatais de risco, com intuito do rastreamento mais eficaz destas condições. Com isso, a pergunta norteadora baseia-se em: Quais os fatores condicionantes ao Pré-Natal de Alto Risco? Sendo objetivo geral de conhecer os fatores condicionantes ao Pré-Natal de Alto Risco, analisando o atendimento do Pré-Natal de Alto Risco e detectando ações de promoção em saúde e prevenção de riscos. 


\section{Metodologia}

Esse estudo é baseado em um método descritivo, sendo uma literatura integrativa, consistido em uma revisão (RIL), subsidiado novos conhecimentos para um problema a ser estudado (Ercole et al. 2014) (Botelho et al., 2011).

Além de ser uma revisão integrativa, também possui um método exploratório qualitativo, portanto, a pesquisa qualitativa é um tipo de pesquisa que busca opiniões sobre pesquisas e classificação exploratória das pesquisas a fim de melhor representar o problema pesquisado e tornar as hipóteses encontradas mais claras (Botelho et al., 2011).

As etapas são: questões norteadoras artigos da literatura nacional, busca dos artigos segundo os critérios de inclusão e exclusão, avaliação, análise e discussão dos resultados.

Os dados inclusão foram artigos de janeiro de 2016 a setembro de 2021, com os descritores português e inglês fornecidos pelo Descritores em Saúde (DECS): Fatores de Risco "Risk Factors" Pré Natal "Pré NatalAcesso aos Serviço de Saúde "Health Services Accessibility". Os critérios de exclusão sendo todos os artigos anteriores ao ano de 2016, Trabalhos de Conclusão de Curso (TCCs), monografias, dissertações, teses, relatos de caso e experiência, além de estudos que estiverem condizentes com o estudo.

A pesquisa foi feita em Bibliotecas Virtuais em Saúde (BVS), sendo elas: a Biblioteca Científica Eletrônica Online (SCIELO), Literatura Latino Americana e do Caribe em Ciências da Saúde (LILACS), usando os termos descritos acima em português e inglês, combinada com o uso do termo booleano AND e sinônimos, use OR encontrando-se Fatores de Risco "Risk Factors" ( $n=695$ ) Pré Natal "Pré Natal" ( $n=603$ ) Acesso aos Serviço de Saúde "Health Services Accessibility" (n=503). O resumo de leitura foi utilizado como ponto de referência inicial para a seleção dos artigos, a fim de determinar, dessa forma, os tópicos de pesquisa e se eles estão incluídos nos parâmetros. A seleção final do artigo foi feita por meio da leitura dos trabalhos previamente selecionados. A coleta de dados é baseada em tabelas, incluindo observações como título, primeiro autor, ano de publicação, tipo de pesquisa, base e conclusões, todas organizadas.

\section{Resultados}

Inicialmente, selecionou-se 111 artigos dentro dos padrões, após isso, somente 80 foram para seleção final a meio da leitura detalhada e avaliação dos critérios previamente estabelecidos, elencado 10 artigos para a construção do trabalho final. Os resultados encontrados no período dos últimos cinco anos (2016 a 2021) de 1.801 artigos, sendo eles da base de dados (LILACS 895) e SCIELO (906) apresentando na Figura 1: 
Figura 1 - Fluxograma do processo de seleção dos artigos da revisão integrativa, 2021.

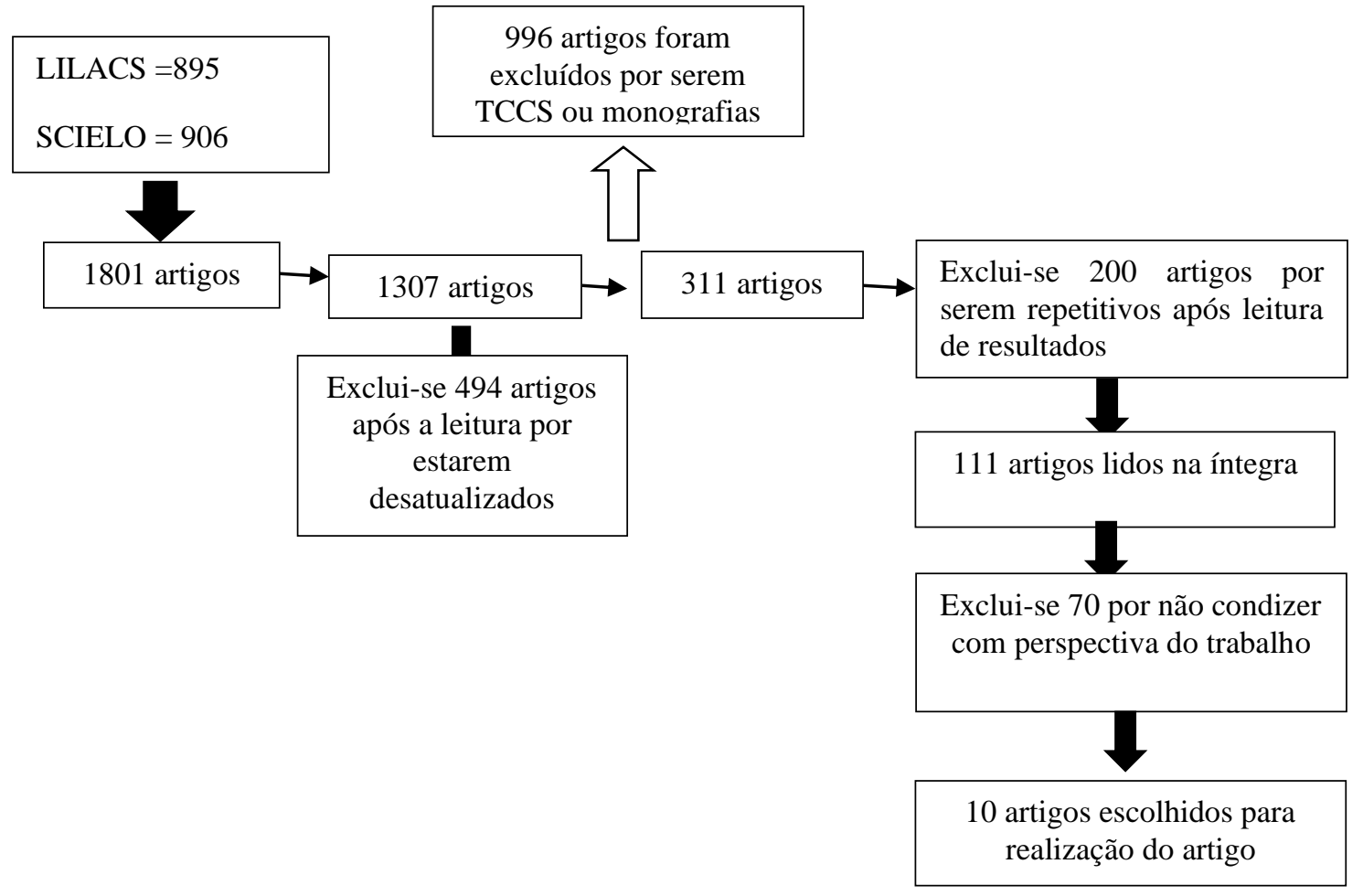

Fonte: Autores (2021).

Com o filtro do idioma (Português 1.051) e (Inglês 750). Foram selecionados 10 artigos na íntegra para discussão desse artigo (Quadro 1) com título, primeiro autor, tipo de estudo, idioma e ano. Para apresentação do artigo dividiu-se em três tópicos para discussão:

Quadro 1 - Apresentação dos estudos incluídos na revisão integrativa, segundo o título, primeiro autor, tipo de estudo, idioma e ano da publicação.

\begin{tabular}{|c|c|c|c|c|}
\hline NOME DO ARTIGO & PRIMEIRO AUTOR & TIPO DE ESTUDO & IDIOMA & ANO \\
\hline $\begin{array}{c}\text { O enfermeiro no pré-natal } \\
\text { de alto risco: papel } \\
\text { profissional }\end{array}$ & $\begin{array}{c}\text { Antonio Rodrigues Ferreira } \\
\text { Junior }\end{array}$ & $\begin{array}{c}\text { Trata-se de um estudo qualitativo } \\
\text { de caráter exploratório descritivo. }\end{array}$ & $\begin{array}{c}\text { PORT } \\
\text { ING }\end{array}$ & 2017 \\
\hline $\begin{array}{c}\text { Síndrome hipertensiva e } \\
\text { resultados perinatais em } \\
\text { gestação de alto risco }\end{array}$ & Marcos Benatti Antunes & $\begin{array}{c}\text { Trata-se de um estudo transversal, } \\
\text { de base documental, com 920 } \\
\text { prontuários de gestantes de um } \\
\text { ambulatório de alto risco. }\end{array}$ & $\begin{array}{c}\text { PORT } \\
\text { ING }\end{array}$ & 2018 \\
\hline $\begin{array}{c}\text { Pré-natal de alto risco em } \\
\text { um serviço de referência: } \\
\text { perfil sociodemográfico e } \\
\text { clínico. }\end{array}$ & $\begin{array}{c}\text { Daniele Socorro de Brito } \\
\text { Souza Paiva }\end{array}$ & $\begin{array}{c}\text { Trata-se de um estudo transversal } \\
\text { retrospectivo e descritivo com } \\
\text { abordagem quantitativa. }\end{array}$ & $\begin{array}{c}\text { PORT } \\
\text { ING }\end{array}$ & 2018 \\
\hline $\begin{array}{c}\text { Indicadores da Qualidade } \\
\text { da Assistência PréNatal de } \\
\text { Alto Risco em uma } \\
\text { Maternidade Pública }\end{array}$ & $\begin{array}{c}\text { Jamiscleia Rodrigues da } \\
\text { Silva }\end{array}$ & $\begin{array}{c}\text { Trata-se de um estudo descritivo, } \\
\text { transversal, quantitativo. }\end{array}$ & PORT & ING \\
\hline
\end{tabular}




\begin{tabular}{|c|c|c|c|c|}
\hline $\begin{array}{l}\text { Gestação de alto risco: } \\
\text { perfil clínico- } \\
\text { epidemiológico das } \\
\text { gestantes atendidas no } \\
\text { serviço de pré-natal da } \\
\text { maternidade pública de } \\
\text { Rio Branco, Acre }\end{array}$ & $\begin{array}{l}\text { Aline Fernanda Silva } \\
\text { Sampaio }\end{array}$ & $\begin{array}{l}\text { Trata-se de um estudo descritivo, } \\
\text { transversal, quantitativo. }\end{array}$ & $\begin{array}{l}\text { PORT } \\
\text { ING }\end{array}$ & 2018 \\
\hline $\begin{array}{l}\text { Perfil sóciodemográfico e } \\
\text { de saúde de gestantes em } \\
\text { um pré-natal de alto risco }\end{array}$ & Juliana Vidal Vieira Guerra & $\begin{array}{c}\text { Trata-se de estudo transversal } \\
\text { descritivo retrospectivo } \\
\text { documental. }\end{array}$ & $\begin{array}{l}\text { PORT } \\
\text { ING }\end{array}$ & 2018 \\
\hline $\begin{array}{l}\text { Estado nutricional materno } \\
\text { e sua associação com o } \\
\text { peso ao nascer em } \\
\text { gestações de alto risco }\end{array}$ & $\begin{array}{c}\text { Alane Cabral Menezes de } \\
\text { Oliveira }\end{array}$ & $\begin{array}{c}\text { Trata-se de estudo transversal } \\
\text { descritivo retrospectivo } \\
\text { documental. }\end{array}$ & $\begin{array}{l}\text { PORT } \\
\text { ING }\end{array}$ & 2018 \\
\hline $\begin{array}{c}\text { Expectativa e satisfação do } \\
\text { acompanhamento pré-natal } \\
\text { em gestantes } \\
\text { de alto risco }\end{array}$ & Fabiana Fontana Medeiros & Trata-se de um estudo qualitativo. & $\begin{array}{l}\text { PORT } \\
\text { ING }\end{array}$ & 2020 \\
\hline $\begin{array}{c}\text { O trabalho do enfermeiro } \\
\text { no pré-natal de alto risco } \\
\text { sob a ótica das } \\
\text { necessidades humanas } \\
\text { básicas }\end{array}$ & $\begin{array}{c}\text { Lívia de Souza Pancrácio } \\
\text { de Errico }\end{array}$ & $\begin{array}{l}\text { Trata-se de estudo transversal } \\
\text { descritivo, qualitativo. }\end{array}$ & $\begin{array}{l}\text { PORT } \\
\text { ING }\end{array}$ & 2017 \\
\hline $\begin{array}{l}\text { Avaliação da atenção à } \\
\text { gestação de alto risco em } \\
\text { quatro metrópoles } \\
\text { brasileiras }\end{array}$ & Juliana Azevedo Fernandes & $\begin{array}{l}\text { Trata-se de estudo avaliativa, de } \\
\text { corte transversal. }\end{array}$ & $\begin{array}{l}\text { PORT } \\
\text { ING }\end{array}$ & 2020 \\
\hline
\end{tabular}

Fonte: Autores (2021).

\section{Discussão}

\section{1 $O$ atendimento ao pré-natal de alto de risco}

As autoras Silva et al. (2018) relatam que o pré-natal constitui como parte fundamental na assistência a gestação para prevenção e detecção de patologias materno-fetal, sendo a responsável por manter bem-estar durante a gravidez. A equipe composta para o pré-natal é multidisciplinar e envolve as várias esferas das Redes de Atenção à Saúde, que devem estar preparadas para as diversidades encontradas, como por exemplo a classificação e diferença entre a Gestação de Baixo e Alto Risco.

Ainda de acordo com os estudos das autoras, o apoio baseia-se em no mínimo 6 de consultas, além dos exames regulares para cada trimestre com os diversos profissionais de saúde, estabelecendo um atendimento holístico. A redução das taxas de mortalidade materna e perinatal estão ligadas a esse serviço, devendo-se atentar ao estado geral da gestante, como clínico, psicológico, socioeconômico e social para descobrimento de manifestações de risco, já que cerca de $10 \%$ a $20 \%$ das gestações pode ser classificada como Gestação de Alto Risco.

As autoras Fernandes et al. (2020) conceituam o Pré-Natal de Alto Risco é aquele que a gestante está sujeita alterações fisiológicas anormais, constituído um problema para acompanhamento da sua gestação e parto, sendo observada nas consultas. A equipe de Estratégia Saúde da Família (ESF) é equipe especializada para classificação de Pré-Natais de Baixo e Alto Risco, sendo um atendimento específico a cada gestante, sendo disponibilizado mapeamento da população de sua abrangência e posteriormente rastreamento dos fatores condicionantes por meio das consultas e visitas domiciliares. Os PréNatais de Alto Risco são encaminhados a unidades obstétricas especificas com intuito de acompanhamento mais de perto para controle das condições encontradas.

As autoras Medeiros et al. (2020) relatam que pela alta complexidade dos casos das gestações de risco, necessita-se de cuidado mais complexo presente no nível secundário e terciário. Sabendo disso, desde 1998, o Ministério da Saúde (MS) criou 
o apoio de Implantação dos Sistemas Estaduais de Referência Hospitalar a Gestante de Alto Risco, com intuito de equiparar pelo princípio da Equidade do Sistema Único de Saúde (SUS) para essas gestantes, tendo um atendimento de acordo com suas necessidades. O projeto tem como objetivo desde sua criação organizar e apoiar a consolidação dos Sistemas de Referência na Área Hospitalar em todo país para público, identificando hospitais ou maternidades para compor este serviço no nível terciário e secundário.

Com isso, cada Estado compõe Sistema Estadual de Referência Hospitalar a Gestante de Alto Risco com dois níveis: secundária, sendo para análise e identificação de risco e responsável pelo encaminhamento a nível terciário de atendimento; a terciaria, disposição de leitos para gestantes de alto risco pela unidade secundaria do sistema. Este atendimento tem as seguintes prioridades: implementar os serviços pré-natais e planejamento familiar especializados; humanização do atendimento com capacitação, treinamento e sensibilização; organização dos comitês de mortalidade materna e neonatal, identificando e investigando os óbitos para intervenção necessária e desenvolvimento dos mecanismos de monitoramento e avaliação permanentes.

Percebe-se que a gestante necessita de um acolhimento integral por especialistas, tendo direito a consultas individuais e grupo, de intervalos regulares até o parto. Esta assistência será principalmente para que as complicações rastreadas sejam sanadas até o nascimento do bebê.

\subsection{Fatores condicionantes ao pré-natal de alto risco}

As autoras Sampaio et al. (2018) conceituam que o período gestacional é a fase de mudanças na mulher, sendo de grande impacto ao corpo, assim existem transformações físicas, emocionais e hormonais, necessitando de ações especificas a cada etapa, existindo fatores intrínsecos a cada perfil. Assim acontece de gestantes terem complicações em suas experiências gravídicas, trazendo riscos à saúde materno-fetal.

Com intuito de diminui essa incidência, as autoras citam que se torna fundamental o diagnóstico clínico das gestantes de Alto Risco, feito pelos profissionais de saúde que necessitam identificar estes dados através do histórico obstétrico tanto atual como anterior e de antecedentes familiares, promovendo os cuidados necessários para binômio mãe e filho.

De acordo Guerra, Valete e Alves (2019), os principais fatores de riscos encontrados estão síndrome hipertensivas, idade avançada ou menoridade, disfunções nutricionais como obesidade ou desnutrição, escolaridade, diabetes mellitus, tromboembolismo e padrões demográficos.

As autoras Paiva et al. (2018) em seu estudo detectaram que as síndromes hipertensivas são um problema sério, determinados por histórico familiar e doença hipertensiva preexistente, apresentando-se na alteração dos valores pressóricos acima de 140/90 mmHg após as 20 semanas, verificando a instabilidade, retirado intercorrências pessoais no dia da aferição. A piora do quadro determina-se em 3 formas pré-eclâmpsia, eclampsia e síndrome de Hellp caracterizada por além de pressão alterada, cefaleia, mialgia e vertigem, sendo por detectada por exames laboratoriais como alteração da proteinúria, trazendo complicações como óbito materno-fetal, risco de prematuridade, convulsões, descolamento de placenta e hemorragia.

Os estudos de Antunes et al. (2017) corroboram destacando que eclampsia pode ser facialmente detectado no prénatal, podendo ser acompanhado e evitado as complicações mais sérias. Assim, Síndromes Hipertensivas Especificas da Gravidez (SHEG) corresponde mais 35\% dos óbitos das gestantes

Os autores Oliveira et al. (2018) constataram que o estado nutricional também constitui como fator condicionante, por esta associado a outras patologias. A obesidade é um fator associado SHEG e diabetes gestacional, além de ameaça de tromboembolismo, levando que $34 \%$ das gestações trazem complicações neonatais importantes como nascimento prematuro e obesidade infantil, necessitando encaminhamento ao nutricionista e orientações necessárias. 
Guerra, Valete e Alves (2019) relatam que a baixa escolaridade é associada ao Pré-Natal de Alto Risco por estabelece a pouca procura do pré-natal e a gravidez indesejada na adolescência, sendo marcada pela vulnerabilidade social, como desemprego e dificuldades econômicas, provocado uma sensibilização a ações e buscativa destas mulheres, principalmente encaminhado aos serviços de saúde especializados.

As autoras detectam que a idade extrema também é um problema, mulheres com menos de 15 anos e idade superior a 38 anos levam erros genéticos mais elevados, sendo relevante. A adolescente torna-se mais problemáticas por não comparecerem ao pré-natal, fazer uso indevido das medicações, além da falta de orientação ao desenvolvimento da criança, mudanças físicas e emocionais da gestação e vias de parto, ademais em destaque o uso de tabagismo, múltiplas gestações e comportamentos sexuais de riscos, necessitando-se que a equipe em saúde rastreie estes dados para acionar os serviços vitais.

É importante ressalta que gestações anteriores podem predispor a fatores de risco, principalmente antecedentes familiares. Medeiros et al. (2020) mostram que $87 \%$ das gestações de risco teve ocorrência de antecedentes familiares de doenças crônicas, constituindo importante princípio. Além de que 50\% das mulheres multíparas apresentam agravos na gestação, mostrando que cesárea se torna um condicionante.

Assim, é notável que estas diversas causas estão associadas a gestação de Alto Risco, necessitando da melhor abordagem, além dos profissionais em saúde dedicarem a identificar tanto precocemente, como prevenir complicações da gestação e puerpério.

\subsection{Ações de enfermagem na promoção a saúde e prevenção de riscos}

Medeiros et al. (2020) descreve que a equipe de enfermagem tem como papel garanti que pré-natal seja de qualidade, prestado assistência humanizada, ouvindo atentamente as queixas, além de avalia histórico gestacional, planejado cuidados e orientado ao atendimento especializado quando necessário.

Assim, a assistência ao pré-natal é necessária para avaliação dos possíveis riscos e diminuição de agravos, assim a classificação acontece a cada consulta, preconizado a gestante no mínimo as 6 consultas, além de acompanhar as gestantes faltosas e realiza buscativa.

No estudo de Enrico et al. (2018) relata que o Ministério da Saúde (MS) preconiza que equipe multiprofissional acompanha essas mulheres, incluindo enfermeiro. A ação da enfermagem destaca-se em planejar e elaboras os cuidados, assim seguindo singularidade da mulher.

A consulta deve ser feita privativa do enfermeiro, tendo que propiciar além do acompanhamento intervenções acerca da importância do pré-natal, amamentação, alimentação e sintomas de perigo. Disto isto, torna-se importante que solicite exames de acordo com protocolo, além do exame físico e avaliação de ultrassons.

Os autores Junior et al. (2017) destacam que as gestantes com diagnóstico de risco devem conduzidas ao serviço de referência e acompanhadas nos dois âmbitos, hospitalar e Atenção Primária, devendo-se atentar na anamnese a informações do histórico obstétrico, sexual e familiar, além de priorizar as necessidades individuais de cada gestante, levando em conta repercussões clinicas, psicológicas e sociais.

Além de olhar físico para os indicadores de peso, pressão arterial, altura uterina e ausculta de BCF, os autores continuam que é notável que se necessita de ações em promoção à saúde, visando que equipe em saúde realize ações em tirar dúvidas de amamentação, desmitificar mitos, atentar-se aos cuidados de higiene intima e das mamas, alertando ao uso das vitaminas no tempo correto e alimentação e ingesta hídrica.

Assim, observa-se que não somente a prevenção de riscos na gestação, fundamente-se que a promoção em saúde deve estar implementando visando acolhimento destas gestantes e um olhar mais singular, assim contribuindo para rastreamento de fatores condicionantes e educação em saúde destas mulheres. 


\section{Considerações Finais}

Tendo em vista o que já foi abordado, fica evidente a importância de conhecer os fatores de risco, sejam físicos, psicológicos ou socioeconômicos, para a vida da equipe multiprofissional, tendo em vista que ela é responsável por rastrear, por meio de busca ativa e ações em saúde por exemplo, identificar através de uma consulta especializada e humanizada, e ajudar no monitoramento e tratamento da situação de saúde da gestante.

Cabe à equipe multiprofissional, em especial a da atenção primária, conhecer os fatores de um pré-natal de alto risco e estar apta a identificar e acompanhar a fim de impedir maiores complicações, tomando ações que priorizem um atendimento especializado e humanizado, visando também a promoção do empoderamento das gestantes através de ações educativas em saúde com o intuito de reduzir as mortes em decorrência de um pré-natal de alto risco.

\section{Referências}

Antunes, M. B., Demitto, M. O., Gravena, A. A. F., Padovani, C., \& Pelloso, S. M. (2017). Síndrome hipertensiva e resultados perinatais em gestações de alto risco. REME. 21, 1-6, 2017.

Barbosa M. I. S., Bosi M. L. M. (2017). Vínculo: um conceito problemático no campo da saúde coletiva. Physis. $27,1003-22$.

Botelho, L. L. R., de Almeida Cunha, C. C., \& Macedo, M. (2011). O método da revisão integrativa nos estudos organizacionais. Gestão e sociedade, 5(11), $121-136$.

Dalla Costa, L., Cura, C. C., Perondi, A. R., França, V. F., \& Bortoloti, D. S. (2016). Perfil epidemiológico de gestantes de alto risco. Cogitare Enfermagem, 21(2).

Domingues R. M. S. M., Viellas E. F., Dias M. A. B., Torres J. A., Theme-Filha M. M., Gama S. G. N. (2015). Adequação da assistência pré-natal segundo as características maternas no Brasil. Rev Panam Salud Pública. 37:140-147

Ercole, F. F., Melo, L. S. D., \& Alcoforado, C. L. G. C. (2014). Revisão integrativa versus revisão sistemática. Revista Mineira de Enfermagem, 18(1), 9-12.

Caldas, D. B., Silva, A. L. R. D., Böing, E., Crepaldi, M. A., \& Custódio, Z. A. D. O. (2013). Atendimento psicológico no pré-natal de alto-risco: a construção de um serviço. Psicologia Hospitalar, 11(1), 66-87.

Campos, C. A. S., Malta, M. B., Neves, P. A. R., Lourenço, B. H., Castro, M. C., \& Cardoso, M. A. (2019). Ganho de peso gestacional, estado nutricional e pressão arterial em gestantes. Revista de saúde publica. 53(8), 1-11.

Junior, A. R. F., de Oliveira Filho, J. T., de Sousa Albuquerque, R. A., Siqueira, D. D. Á., Rocha, F. A. A., \& Rodrigues, M. E. N. G. (2017). O enfermeiro no pré-natal de alto risco: papel profissional. Revista Baiana de Saúde Pública. 41(3) p. 650-667.

Fernandes, J. A., Venâncio, S. I., Pasche, D. F., Silva, F. L. G. D., Aratani, N., Tanaka, O. Y. \& Campos, G. W. D. S. (2020). Avaliação da atenção à gestação de alto risco em quatro metrópoles brasileiras. Cadernos de Saúde Pública. 36(5), p. 1-14, 2020.

Errico, L. D. S. P. D, Bicalho, P. G, Oliveira, T. C. F. L. D, \& Martins, E. F. (2018). O trabalho do enfermeiro no pré-natal de alto risco sob a ótica das necessidades humanas básicas. Revista Brasileira de Enfermagem .71, p. 1257-1264.

Guerra, J. V. V., Valete, C. O. S., \& Alves, V. H. (2019). Perfil sóciodemográfico e de saúde de gestantes em um pré-natal de alto risco. Brazilian Journal of Health Review, 2(1), 249-261.

Medeiros, F. F., Lourenço, J. C., Rodrigues, M. H., Ferrari, R. A. P., Serafim, D., \& Cardelli, A. A. M. (2020). Expectativa e satisfação do acompanhamento pré-natal em gestantes de alto risco. Revista Eletrônica Acervo Saúde. 40(1), p. 1-8.

Paiva, D. S. D. B. S., de Moura Nunes, H. H., da Silva Moreira, S. F., \& Ferreira, M. G. S. (2019). Pré-natal de alto risco em um serviço de referência: perfil sociodemográfico e clínico. Revista Eletrônica Acervo Saúde, 11(2), 1-12.

Sanine PR, Venâncio SI, Silva FLG, Aratani N, Moita MLG \& Tanaka OY. (2019). Atenção ao pré-natal de gestantes de risco e fatores associados no município de São Paulo. Cad Saúde Pública. 35:e00103118.

Sánchez-Gómez A., Cevallos W., Grijalva M. J., Silva-Ayçaguer L. C., Tamayo S., Jacobson J. O., et al. (2016). Factores sociales asociados con la utilización de los servicios de atención prenatal en Ecuador. Rev Panam Salud Pública. 40:341-346.

Saude, M. (2012) Série A. normas e manuais técnicos: Gestação de Alto Risco. Ministério da Saúde.

Saude, M. (2017). Rastreamento e diagnóstico de diabetes mellitus gestacional no brasil. Ministério da Saúde.

Saude, M. Secretaria Executiva. (2001). Gestante de alto risco. Ministério da Saúde.

Sampaio, A. F. S., Rocha, M. J. F. D., \& Leal, E. A. S. (2018). Gestação de alto risco: perfil clínico-epidemiológico de gestantes atendidas no serviço de prénatal da Maternidade Pública de Rio Branco, Acre. Revista Brasileira de Saúde Materno Infantil. 18(3), 559-566. 
Research, Society and Development, v. 10, n. 15, e509101522922, 2021

(CC BY 4.0) | ISSN 2525-3409 | DOI: http://dx.doi.org/10.33448/rsd-v10i15.22922

Silva, J. R., De Oliveira, M. B. T., Santos, F. R. P., Neto, M. S., Ferreira, A. G. N., \& Santos, F. S. (2018). Indicadores da qualidade da assistência pré-natal de alto risco em uma maternidade pública. Revista Brasileira de Ciências da Saúde, 22(2), 109-116.

Oliveira, A. C. M. D., Pereira, L. A., Ferreira, R. C., \& Clemente, A. P. G. (2018). Estado nutricional materno e sua associação com o peso ao nascer em gestações de alto risco. Ciência \& Saúde Coletiva. 23(7), p.2373-2382. 